\title{
Ein Leben lang unsere Gesundheit fördern: Gesundheitsförderung im Lebensverlauf
}

\author{
Die 16. Nationale Gesundheitsförderungs-Konferenz findet am 29. Januar 2015 in \\ Luzern statt. Die Referierenden werden ihre neusten Ergebnisse im Zusammenhang \\ mit der Lebenslaufforschung vorstellen sowie deren Folgen für die Gesundheitsförde- \\ rung und Prävention. Wie wirken sich Übergangsperioden im Alterungsprozess und \\ kritische Ereignisse auf die Gesundheit aus? Welche Mechanismen führen in den ein- \\ zelnen Lebensabschnitten zu Anfälligkeiten und Ungleichheiten?
}

Barbara Weil ,

Catherine Favre Kruit ${ }^{b}$

a FMH, Abteilung Gesundheitsförderung und Prävention

b Gesundheitsförderung Schweiz

1 Nationaler Forschungspo LIVES: www.lives-nccr.ch, Transitions from Education to Employment (TREE): tree.unibas.ch, Frühe Förderung ab Geburt Zeppelin 0-3: www.zeppelin-hfh.ch, z-proso: www.z-proso.ethz.ch

Korrespondenz: Barbara Weil FMH / Abteilung Gesundheitsförderung und Prävention Elfenstrasse 18

CH-3000 Bern 15

Tel. 0313591111

Fax 0313591112
Unser gegenwärtiges Umfeld ist geprägt von der Alterung der Bevölkerung, einem Anstieg der chronischen Krankheiten und ständig steigenden Gesundheitskosten - Entwicklungen, die unsere Gesellschaft und unser Gesundheitssystem vor grosse Herausforderungen stellen.

Nur ein disziplinen- und sektorenübergreifender Ansatz kann helfen, Lösungen auf dem Gebiet der Gesundheitsförderung und Prävention aufzuzeigen. Der Lebenslauf-Ansatz ist ein solcher. Er setzt an der Schnittstelle zwischen öffentlicher Gesundheit, Psychologie, Soziologie, Demographie und Biologie sowie Geschichte und Wirtschaft an und ergänzt damit einen rein auf Gesundheitsdeterminanten basierenden Ansatz. Ziel ist, die Zusammenhänge und Wechselwirkungen besser zu verstehen, die in den verschiedenen Lebensphasen zwischen dem Menschen und seinem Umfeld bestehen, und zu ergründen, wie sich diese auf die Gesundheit auswirken.

Unsere Gesundheit von morgen ist abhängig von unseren Erfahrungen und unserem sozialen, ökonomischen und ökologischen Kontext von heute. Die frühe Kindheit, einschliesslich der pränatalen Phase und der Gesundheit unserer Mutter vor der Schwangerschaft, spielt eine entscheidende Rolle für unsere künftige Gesundheit. Jedes einschneidende Ereignis wirkt sich auf unsere Gesundheit aus. Dies gilt besonders für Geschehnisse in kritischen Phasen oder in Zeiten des Übergangs wie dem Beginn der Schulzeit und der Adoleszenz, dem Eintritt ins Berufsleben, der Elternschaft oder der Pensionierung. Diese einschneidenden Lebenserfahrungen, seien sie nun positiv oder negativ, haben einen kumulierenden Effekt. Während das Wechselspiel negativer Faktoren zu einer Steigerung von Gesundheitsproblemen führt, können positive Faktoren die negativen Auswirkungen vorangegangener oder folgender Lebensetappen verringern. Wir sind ein Leben lang Risikofaktoren ausgesetzt, die unsere Gesundheit beeinträchtigen, ebenso aber auch schützenden Faktoren, die unserer Gesundheit zugutekommen. Diese Risiko- und Schutzfaktoren sind jedoch nicht auf das
Verhalten des Einzelnen beschränkt, sondern auch abhängig vom familiären Kontext, von der Nachbarschaft, von der Gemeinschaft, vom beruflichen Umfeld und nicht zuletzt von der Sozialpolitik.

Um den Lebenslaufansatz anschaulicher und fassbarer zu machen, haben wir Fachleute eingeladen, die uns die Ergebnisse ihrer Forschungsarbeiten auf dem Gebiet der frühen Kindheit, der Adoleszenz, des Berufslebens und des Alters vorstellen [1]. Sie liefern interessante Ergebnisse welche zeigen, dass eine auf den Lebenslaufansatz ausgerichtete Gesundheitsförderungs- und Präventionspolitik ein koordiniertes Vorgehen der Akteure im Bereich der Prävention, des Gesundheitswesens, der psychosozialen, medizinischen sowie sozialen Betreuung bedingt. Ebenfalls miteinbezogen werden müssen Gesundheitsdeterminanten wie die Hygiene, der Lebensstil, die Erziehung, das berufliche Umfeld und die Umwelt.

Wenn auch der Lebenslauf einer Person einzigartig ist, so können dennoch auf der Ebene Gesamtbevölkerung Lebenslauf-Typologien im Vornherein ausfindig gemacht und entwickelt werden. Dadurch können die nötigen Ressourcen antizipiert werden, um zukünftige Bedürfnisse abzudecken. Das Ziel eines solchen integrierten und multidisziplinären Ansatzes besteht darin, das Gesundheitssystem effizienter zu gestalten. Dabei ist zu gewährleisten, dass die Bevölkerung Zugang zu guten Dienstleistungen und Beratungen erhält, welche von professionellen Fachleuten in zweckmässigen Strukturen zum richtigen Moment und zu niedrigen Kosten erbracht werden.

\section{Nationale Gesundheitsförderungs- Konferenz: Ein Leben lang unsere Gesundheit fördern \\ Donnerstag, 29. Januar, Messe Luzern, organisiert in Zusammenarbeit und mit der Unterstützung des Kantons Luzern.}

Programm und Anmeldung:

www.gesundheitsfoerderung.ch/konferenz 